\title{
Relation between Surface Relaxation and Surface Force Constants in Clean and Oxygen-Covered $\mathrm{Ni(001)}$
}

\author{
Joost W. M. Frenken and J. F. van der Veen \\ FOM-Institute for Atomic and Molecular Physics, 1098 SJ Amsterdam, The Netherlands \\ and \\ G. Allan \\ Laboratoire de Physique des Solides, Institut Supérieur d'Electronique du Nord, F-59046 Lille Cédex, France
} (Received 6 June 1983)

\begin{abstract}
Ion shadowing and blocking measurements show that the first interlayer spacing of $\mathrm{Ni}(001)$ is contracted if the surface is clean, but expanded if it is covered by half a monolayer of oxygen. The thermal vibration amplitude of surface atoms is strongly enhanced. A simple tight-binding model relates the change in first interlayer spacing to a change in surface force constants, and explains the recently measured surface phonon dispersion curves.
\end{abstract}

PACS numbers: $68.20 \_t, 68.30 \_+z$

It has been proposed that the dynamical properties of surfaces are sensitively dependent on structural changes such as surface relaxation and reconstruction, defects, and chemisorption. Clearly, a detailed investigation of the changes in interatomic distances and vibrational properties induced at a surface by, e.g., oxygen chemisorption will be extremely valuable, since it will ultimately provide the clues to understanding phenomena like surface catalytical activity, or the transition from chemisorption to oxidation.

In this Letter we present new experimental data on relaxation and vibration amplitudes of surface atoms in $\mathrm{Ni}(001)$. For the clean surface the first interlayer spacing is contracted by $-3.2 \%$, but chemisorption of oxygen induces a substantial outward relaxation, resulting in a $+5.2 \%$ expansion at $\frac{1}{2}$ monolayer coverage. Using a tightbinding model we show that these relaxations imply for the clean surface a strengthening of the surface interlayer force constants with respect to their crystalline values, and for the oxygencovered surface a substantial weakening. The modified force constants derived from this model bring theoretical vibration amplitudes and frequencies in remarkable agreement with measured amplitudes and with the frequencies recently measured in inelastic electron-scattering experiments. ${ }^{1,2}$

The experiments will only be discussed briefly here. We have used the technique of Rutherford backscattering (RBS) in shadowing and blocking geometry, which has been described by Saris. ${ }^{3}$ The experimental setup features a novel toroidal energy analyzer of backscattered ions, allowing measurement of surface blocking and bulk blocking angles with a precision of $\pm 0.03^{\circ}$ 。 $\mathrm{Ni}(001)$ surfaces were prepared by standard procedures. A well ordered $c(2 \times 2)$ oxygen overlayer was produced by exposing the clean surface to 20 langmuirs of $\mathrm{O}_{2}$ (1 langmuir $=10^{-6}$ Torr sec) at $400 \mathrm{~K}$. Measurements were carried out at $370 \mathrm{~K}$. From the area of the oxygen peak in the backscattering spectra the oxygen coverage for the $c(2 \times 2)$ surface was determined to be $\theta=0.46 \pm 0.04$ monolayer $\left(1\right.$ monolayer $\equiv 1.6 \times 10^{15}$ atoms $\left./ \mathrm{cm}^{2}\right)$.

A parallel $52-\mathrm{keV}$ proton beam was aligned with the [301] bulk axis of the crystal (Fig. 1). In this scattering geometry, atoms in the third and deeper layers of the crystal are almost completely shadowed by the atoms in the first two layers. Nickel atoms in the second layer, however, have a nonnegligible hitting probability, which depends sensitively on the surface root mean square (rms) thermal displacement. Protons backscattered from a second-layer nickel atom can leave the crystal in all directions except those in which they are blocked by a first-layer atom on their way out. In these surface blocking directions, the second-layer contribution to the surface backscattering signal is strongly reduced. From the exact angles at which these minima occur, the first interlayer spacing can be determined in

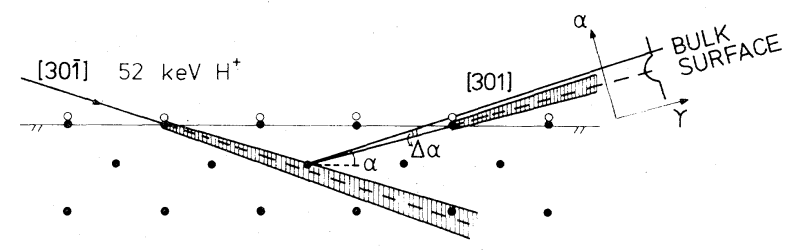

FIG. 1. Scattering geometry in the (010) plane perpendicular to the (001) surface. The angular shift $\Delta \alpha$ is indicated for a contracted surface. 
principle by simple trigonometry. ${ }^{4}$

Figure 2 shows such surface blocking minima for clean and $c(2 \times 2)$ oxygen-covered $\mathrm{Ni}(001)$. These measurements have been divided by the angular-dependent part of the Rutherford cross section, and calibrated $( \pm 3 \%)$ to give the number of nickel layers visible to both ion beam and detector, as a function of exit angle $\alpha$ with respect to the surface plane. The vertical lines in Fig. 2 indicate the (measured) positions of the bulk axes. The surface blocking minima for both the clean and the $c(2 \times 2) \mathrm{O}$ surface are shifted with respect to the [301] bulk axis. The shift to lower exit angles for the clean surface $\left(\Delta \alpha=-0.75^{\circ}\right)$ corresponds to $\mathrm{a}-(3.2 \pm 0.5) \%(=-0.056 \pm 0.008$ $\AA$ ) contraction of the spacing between the first two nickel layers with respect to the bulk interlayer spacing of $1.76 \AA,^{5}$ in disagreement with the expansion found in low-energy electron diffraction (LEED) and spin-polarized LEED studies. ${ }^{6}$ The shift to higher exit angles for the $c(2 \times 2) \mathrm{O}$ surface $\left(\Delta \alpha=+0.97^{\circ}\right)$ indicates $\mathrm{a}+(5.2 \pm 1.5) \%$ $(=0.09 \pm 0.03 \AA)$ expansion of the first interlayer spacing with respect to the bulk spacing. ${ }^{5,7}$

Surface rms displacements have been obtained by comparing the data with Monte Carlo simulations of the experiment. ${ }^{8}$. The fitting parameter in these simulations was the two-dimensional

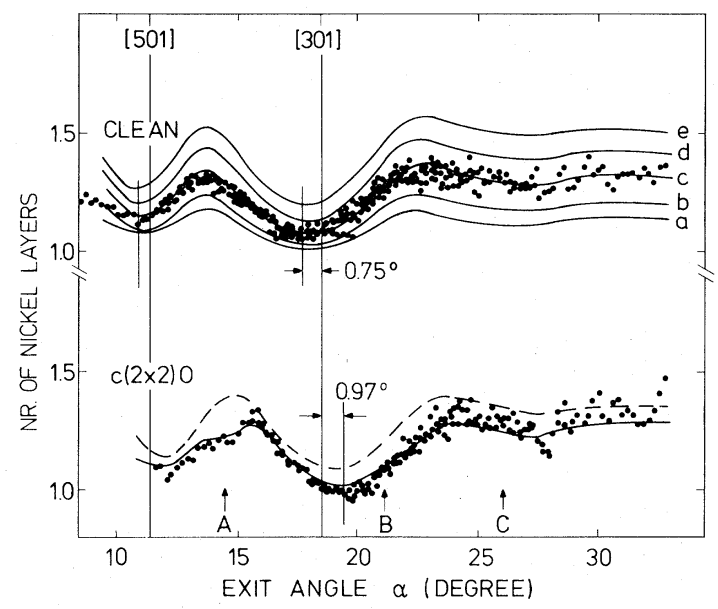

FIG. 2. Blocking curves for Ni(001). Solid curves represent computer simulations with relaxation values of $-3.2 \%$ for the clean and $+5.2 \%$ for the $c(2 \times 2) \mathrm{O}$ surface. (a) $\rho_{12}=0.16 \AA$ (bulk vibrations), (b) $\rho_{12}$ $=0.18 \AA$, (c) $\rho_{12}=0.20 \AA$, (d) $\rho_{12}=0.23 \AA$, (e) $\rho_{12}$ $=0.25 \AA$. In the simulations for the $c(2 \times 2) \mathrm{O}$ surface $\rho_{12}=0.22 \AA$ and $\sigma_{\text {ox }}=0.13 \AA$ were used. Removing the oxygen atoms without changing the parameters for the nickel substrate results in the dashed curve. $A, B$, and $C$ indicate oxygen blocking directions.
(2D) rms relative displacement of first and second layer atoms,

$$
\rho_{12}=\left[\left(\sigma_{1 \perp}^{2}+\sigma_{2 \perp}^{2}\right) \cos ^{2} \alpha+\left(\sigma_{1 \|}^{2}+\sigma_{2 \|}^{2}\right)\left(1+\sin ^{2} \alpha\right)\right]^{1 / 2},
$$

where the $\sigma$ 's are 1D rms displacements of firstand second-layer atoms perpendicular and parallel to the surface, and $\alpha=18.4^{\circ}$ is the angle between ion beam or detector and surface plane. Third- and deeper-layer atoms were given a 1D rms displacement of $\sigma_{\text {bulk }}=0.08 \AA$. Simulations for $\rho_{12}$ ranging from $0.16 \AA$ to $0.25 \AA$ are shown in Fig. 2 for the clean surface. Excellent agreement with the measured blocking curve over the entire range of exit angles is obtained for $\rho_{12}$ $=0.20 \AA$ 。 A least-squares comparison of simulations and measurements yields a best-fit value of $\rho_{12}=0.20 \pm 0.01 \AA$. For the $c(2 \times 2)$ O blocking curve the best fit is obtained for a value of $\rho_{12}=0.22$ $\pm 0.02 \AA$ for the $2 \mathrm{D}$ rms relative displacement of the first two nickel layers.

The additional blocking of emerging ions by oxygen in the $c(2 \times 2) \mathrm{O}$ blocking curve has enabled us to determine the position of the adsorbed oxygen atoms, and to estimate their 1D rms displacement $\sigma_{\text {ox }}$. The sharp blocking feature $(A)$ at $14.4^{\circ}$ in Fig. 2 is caused by oxygen atoms, residing in (or near ${ }^{9}$ ) the fourfold hollow sites of the $\mathrm{Ni}(001)$ surface, blocking off protons backscattered from second-layer nickel atoms. The oxygen atoms must be $2.71 \pm 0.10 \AA$ above the second nickel layer to give rise to a blocking dip at this angle. Subtracting the (expanded) first interlayer spacing gives a value of $0.86 \pm 0.10 \AA$ for the distance between the oxygen atoms and the first nickel plane, in excellent agreement with other experimental evidence ${ }^{10}$ and excluding the possibility of an "oxidic" position ${ }^{11}$ at $0.26 \AA$. The correct amount of oxygen blocking was obtained for a $1 \mathrm{D}$ rms displacement of $\sigma_{\text {ox }}=0.13 \AA$.

At intermediate oxygen coverages of $\theta=0.27$ \pm 0.04 and $\theta=0.37 \pm 0.04$ monolayer, the relaxation values were determined to be $+(2.0 \pm 1.0) \%$ and $+(3.2 \pm 1.5) \%$, respectively.

The theoretical model we have used to calculate the structure and dynamics of the clean and the oxygen-covered $\mathrm{Ni}(001)$ surfaces has been discussed elsewhere ${ }^{12}$ and will be outlined concisely. The nickel $a$-band density of states is described within the tight-binding approximation. The attractive part of the cohesive energy is evaluated from the first two moments of the density of states, which are expressed in terms of twocenter resonance integrals between $d$ atomic orbitals. As these integrals depend primarily on 
the distance $R_{i j}$ between atoms $i$ and $j,{ }^{12}$ we take a mean value $\beta_{i j}\left(R_{i j}\right)$ of the true resonance integrals between nearest neighbors:

$$
\beta_{i j}\left(R_{i j}\right)=\beta_{i j}{ }^{0} \exp \left(-q_{i j} R_{i j}\right) .
$$

The potential accompanying the small charge transfer between atoms in the surface region, which must be self-consistently determined, is approximated rather well by demanding every atom to be neutral. ${ }^{12}$ The repulsive part of the cohesive energy is represented by a Born-Mayer interatomic potential

$$
C_{i j}\left(R_{i j}\right)=C_{i j}{ }^{0} \exp \left(-p_{i j} R_{i j}\right) .
$$

For the clean surface our model has four parameters, which have been fitted to the bulk interatomic distance, the bulk phonon dispersion curves, and the bulk cohesive energy. Best-fit values are

$$
\begin{aligned}
& \beta_{\mathrm{Ni}-\mathrm{Ni}}^{0}=14.2 \mathrm{eV}, \quad C_{\mathrm{Ni}-\mathrm{Ni}}^{0}=2740 \mathrm{eV}, \\
& p_{\mathrm{Ni}-\mathrm{Ni}}=4.10 \AA^{-1}, \quad \text { and } q_{\mathrm{Ni}-\mathrm{Ni}}=0.884 \AA^{-1} .
\end{aligned}
$$

A second-order expansion of the cohesive energy with respect to atomic positions is then used to minimize the energy and to find the dynamical matrix. This yields a $-3.2 \%$ surface contraction, in remarkable accordance with our measurements. The contraction is responsible for an increase of the force constant between first- and second-layer nickel atoms to 1.20 times the bulk nearest-neighbor force constant. The surface phonon frequencies that we obtained from a $20-$ layer slab calculation are shown in Fig. 3. Only modes which are even with respect to the (110) plane have been included. The calculated $S_{4}$ mode agrees well with the dispersion curve measured by Lehwald et al. ${ }^{1}$ This shows that angle-bending forces, proposed by these authors as a possible explanation for the somewhat high frequencies of the $S_{4}$ mode near the $\bar{X}$ symmetry point, need not be invoked, since the stiffening of the surface, required for such high frequencies, is a result of the surface contraction. Vibration amplitudes and correlation coefficients ${ }^{13}$ for surface atoms were calculated with the continued-fraction method. ${ }^{14}$ This yielded

$$
\begin{aligned}
& \sigma_{1 \perp}=0.102 \AA, \quad \sigma_{1 \|}=0.097 \AA, \\
& \sigma_{2 \perp}=0.090 \AA, \quad \text { and } \sigma_{2 \|}=0.084 \AA .
\end{aligned}
$$

The corresponding value of $\rho_{12}=0.19 \AA$ agrees very well with the experimental value of $\rho_{12}=0.20$ $\pm 0.01 \AA$.

The $c(2 \times 2)$ oxygen overlayer introduces four

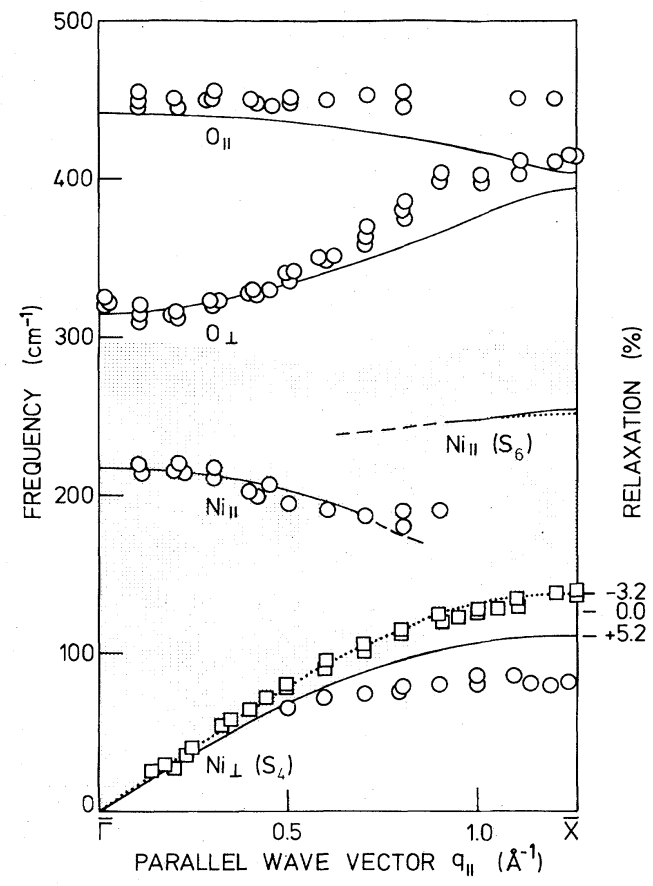

FIG. 3. Calculated surface phonon dispersions for clean (dotted curves) and oxygen-covered (full curves) $\mathrm{Ni}(001)$, along the $\bar{\Gamma}-\bar{X}$ direction of the substrate Brillouin zone. The shaded region corresponds to projected bulk phonon bands. Experimental data for clean (squares) and oxygen-covered (circles) $\mathrm{Ni}(001)$ are from Refs. 1 and 2 , respectively.

extra parameters in our model:

$$
\beta_{\mathrm{Ni}-\mathrm{O}}{ }^{0}, C_{\mathrm{Ni}-\mathrm{O}}{ }^{0}, p_{\mathrm{Ni}-\mathrm{O}}, \text { and } q_{\mathrm{Ni}-\mathrm{O}} \text {. }
$$

When we choose combinations of these parameters that yield the correct $\mathrm{Ni}(001) c(2 \times 2) \mathrm{O}$ surface structure (i.e., $+5.2 \%$ surface expansion and oxygen atoms in the fourfold hollow sites at a height of $0.86 \AA$ ), only two degrees of freedom are left. These can be further reduced to only one degree of freedom if we require one of the adatom frequencies (e.g., the frequency for the perpendicular motion of oxygen at the $\bar{\Gamma}$ point) to reproduce the measured value. ${ }^{2}$ Within the remaining set of allowed combinations for the four $\mathrm{Ni}-\mathrm{O}$ parameters, all calculated oxygen and nickel surface vibration frequencies are constant to within a few inverse centimeters (details of this procedure will be published elsewhere). Figure 3 shows the resulting phonon dispersion curves. The substantial decrease of the $S_{4}$ frequency at the $\bar{X}$ point is a result of a reduction of the firstto second-nickel-layer force constant to $70 \%$ of the bulk value, accompanying the $+5.2 \%$ surface expansion. Very recently, a downward frequency 
shift has indeed been observed by Szeftel et al. ${ }^{2}$ Apart from an apparent discrepancy in the magnitude of the $S_{4}$ frequency shift, the calculated phonon dispersion curves are in good agreement with those data. ${ }^{15}$ The $1 \mathrm{D} \mathrm{rms}$ displacements of surface nickel atoms were calculated to be $\sigma_{1 \perp}=0.12$ $\AA, \sigma_{1 \|}=0.076 \AA$ in the direction of adsorbed oxygen atoms and $0.094 \AA$ perpendicular to such directions, $\sigma_{2 \perp}=0.085 \AA$, and $\sigma_{2 \|}=0.081 \AA$, corresponding to $\rho_{12}=0.19 \AA$. This value is close to the experimental value of $\rho_{12}=0.22 \pm 0.02 \AA$. For the oxygen atoms the calculation yielded $\sigma_{\text {ox } \perp}=0.11$ $\AA$ and $\sigma_{\text {oxll }}=0.09 \AA$, slightly lower than the experimental estimate of $\sigma_{o x}=0.13 \AA$.

In summary, the clean $\mathrm{Ni}(001)$ surface is contracted, and consequently stiffened. Oxygen chemisorption induces a surface expansion, accompanied by a substantial weakening of the force constants between first- and second-layer nickel atoms. Further studies on various metal-oxygen systems are required to establish whether or not a weakened metal interlayer bond strength is a necessary precursor state for penetration of oxygen into the surface, i.e., for oxidation.

This work is sponsored by Fundamenteel Onderzoek der Materie with financial support from Nederlandse Organisatie Voor Zuiver-Wetenschappelijk Onderzoek.

${ }^{1}$ S. Lehwald, J. M. Szeftel, H. Ibach, T. S. Rahman, and D. L. Mills, Phys. Rev. Lett. 50, 518 (1983).

${ }^{2}$ J. M. Szeftel, S. Lehwald, H. Ibach, T. S. Rahman, J. E. Black, and D. L. Mills, Phys. Rev. Lett. 51, 268 (1983).

${ }^{3}$ F. W. Saris, Nucl. Instrum. Methods 194, 625 (1982). ${ }^{4}$ Surface blocking angles are corrected for the nonzero impact parameter for backscattering, and for the Rutherford cross section.

${ }^{5}$ The change in spacing between second and third layers is less than $1 \%$ (to be published).

${ }^{6} \mathrm{~J}$. E. Demuth, P. M. Marcus, and D. W. Jepsen, Phys. Rev. B 11, 1460 (1975); R. Feder, S. F. Alvarado, E. Tamura, and E. Kisker, Surf. Sci. 127, 83 (1983).

${ }^{7} \mathrm{~A}$ similar expansion has been observed for oxygen on Ni(111); T. Narusawa, W. M. Gibson, and E. Törnqvist, Phys. Rev. Lett. $\underline{47}, 417$ (1981).

${ }^{8}$ In the simulations a Molière scattering potential has been used: G. Molière, Z. Naturforsch. A2, 133 (1947).

${ }^{9}$ J. E. Demuth, N. J. Dinardo, and G. S. Cargill, Phys. Rev. Lett. 50, 1373 (1983).

${ }^{10}$ For example, J. Stöhr, R. Jaeger, and T. Kendelewicz, Phys. Rev. Lett. 49, 142 (1982).

${ }^{11}$ T. H. Upton and W. A. Goddard, Phys. Rev. Lett. $\underline{46}$, 1635 (1981).

${ }^{12}$ G. Allan and J. Lopez, Surf. Sci. $\underline{95}, 214$ (1980), and references therein.

${ }^{13}$ Correlations were found to be negligible for the geometry of Fig. 1.

${ }^{14}$ J. E. Black, B. Laks, and D. L. Mills, Phys. Rev. B 22, 1818 (1980), and references therein.

${ }^{15}$ Apart from pure bulk and surface modes, the slab calculation also yielded several weak modes of mixed character. One of these (starting from $218 \mathrm{~cm}^{-1}$ at $\bar{\Gamma}$ ) has been observed experimentally, and has therefore been included in Fig. 3 . 